\title{
Middle pancreatectomy for selected pancreatic neck tumours
}

\author{
P. J. Lakhey, B. Ghimire, M. Khakurel. \\ Gastrointestinal Surgery Unit, Department of Surgery, Tribhuwan University Teaching Hospital, Kathmandu, Nepal. \\ Correspondence to: Dr Paleswan Joshi Lakhey, MCh Resident, Gastrointestinal Surgery Unit, Department of Surgery, \\ Tribhuwan University Teaching Hospital, Kathmandu, Nepal.
}

Email:lakheypj@gmail.com

\begin{abstract}
Middle pancreatectomy has been indicated for small benign lesions located in the neck or body of the pancreas. The proponents of middle pancreatectomy argue that it preserves pancreatic exocrine and endocrine function thus avoiding pancreatic exocrine insufficiency and diabetes, and preserves spleen thereby avoiding life-threatening complication of post splenectomy sepsis. However, others express concern regarding the increased risk of pancreatic fistula and oncological adequacy. Here, we report two cases with low grade cystic neoplasm involving the neck of the pancreas that underwent middle pancreatectomy with variable outcome.
\end{abstract}

\section{Introduction}

Middle pancreatectomy is a surgical procedure that removes the middle segment of the pancreas and preserves the distal pancreas and spleen. This procedure has been described using various terms such as "central pancreatectomy," "middle segment pancreatectomy," "segmental pancreatectomy," and "intermediate pancreatectomy." 1,2

It was Letton and Wilson in 1959 who reported two cases of traumatic midpancreatic transection followed by a reconstruction with a Roux-en-Y jejunal loop anastomosed to the distal part of the gland. ${ }^{3}$ Later in 1984, Dagradi and Serio, reported middle pancreatectomy with an "oncological" indication, treating a pancreatic insulinoma. However, this was later popularised by Serio and Iacono and the procedure has been named as the Dagradi-Serio-Iacono operation. ${ }^{4}$

Usually the tumours of head of the pancreas are treated by pancreaticoduodenectomy and that of body and tail by distal pancreatectomy. It the tumours in the neck and adjacent body of the pancreas that pose the real challenge, as extensive pancreatic resection is associated with impairment of both endocrine and exocrine function. Though the tumours up to 2 centimetres $(\mathrm{cm})$ in size are managed by enucleation, there is always risk of injury to the pancreatic duct. The proponents of middle pancreatectomy argue that it preserves pancreatic exocrine and endocrine function thus avoiding pancreatic exocrine insufficiency and diabetes, and preserves spleen thereby avoiding life-threatening complication of post splenectomy sepsis. However, others express concern regarding the increased risk of pancreatic fistula and oncological adequacy. ${ }^{5}$ The literature provides enough evidence that middle pancreatectomy is a safe and feasible procedure for benign or borderline lesions of pancreas situated in the neck of the pancreas. Here, we report two cases of pancreatic neck tumors that were managed with middle pancreatectomy.

\section{Case report}

Case 1: A 42 year old lady without any co morbidity presented with vague abdominal pain without any weight loss or loss of appetite or other gastrointestinal symptoms. Clinical exam did not reveal any positive findings. Her abdominal ultrasound revealed inhomogeneous mass in the neck of the pancreas suggesting focal pancreatitis. Contrast enhanced CT scan of abdomen was suggestive of solid pseudo papillary tumor of pancreas. She underwent middle pancreatectomy, with pancreaticojejunostomy of the distal pancreatic stump by invagination technique (Fig. 1). The proximal part of pancreatic transection was done with a GIA stapler. There was $3.5 \times 2.5 \times 3 \mathrm{~cm}$ solid cystic mass in the 
neck of the pancreas. The final histopathology report was solid pseudo papillary tumor of pancreas. Unfortunately, the patient developed grade $\mathrm{C}$ pancreatic fistula from the proximal stump where the stapler had been applied. She developed postpancreatectomy hemorrhage and intraabdominal abscess. She succumbed due to pneumonia, ARDS and multiorgan dysfunction syndrome.

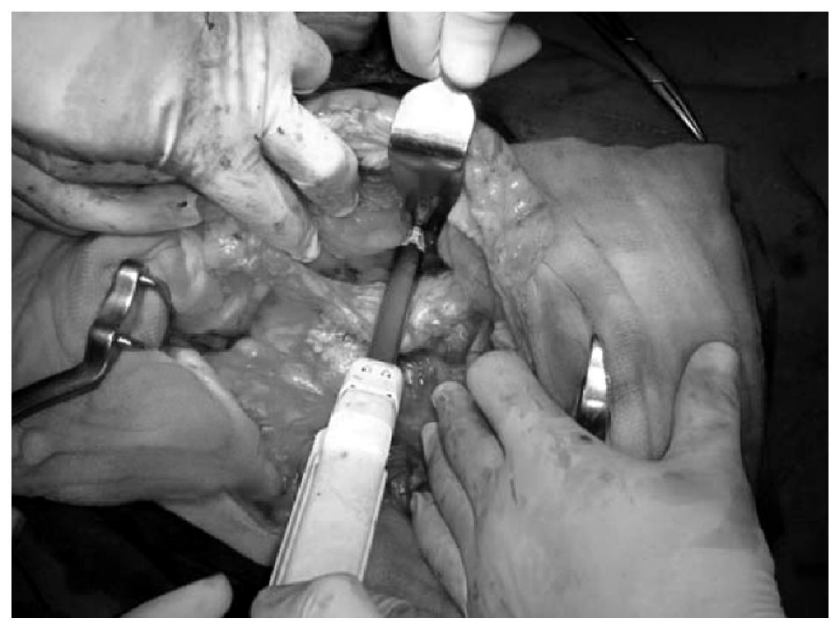

Fig. 1: pancreatic transection with a GIA stapler

Case 2: A 46 year old lady without any co morbidity presented with epigastric pain and burning sensation which radiated to the back. The pain was aggravated by meals and was associated with loss of weight and appetite. The clinical exam was normal. The abdominal ultrasound revealed $2.5 \times 1.7 \mathrm{~cm}$ irregular thick wall cystic mass with calcification in the head of the pancreas. CECT abdomen revealed nonenhancing cystic mass with foci of eccentric calcification suggestive of microcystic serous adenoma in the head of the pancreas. Thus Whipple's operation was planned. However, as the tumour was in the neck of the pancreas, she underwent middle pancreatectomy with the proximal pancreatic duct occluded with suture and the distal pancreatic stump was fashioned into pancreaticojejunostomy by invagination technique. There was $4 \times 3 \mathrm{~cm}$ multiloculated cystic mass in the neck of the pancreas. The final histopathology report revealed serous oligocystic adenoma. Her postoperative period was uneventful and was discharged in the tenth postoperative day.

\section{Discussion}

Middle pancreatectomy has been added to the armamentarium of pancreatic surgery as a safe and feasible procedure for selected tumours of the neck of the pancreas. The prerequisite for middle pancreatectomy has been described as a small lesion less than $5 \mathrm{~cm}$ in diameter, benign or low grade malignant tumours, and distal stump at least 5 $\mathrm{cm}$ in length. ${ }^{6}$ Both of our patients fulfilled the criteria and were subjected to middle pancreatectomy. There have been concern regarding the oncological adequacy of this surgery and frozen section is said to be mandatory as sometimes it is difficult to distinguish benign lesions from malignant lesions preoperatively.

Middle pancreatectomy preserves the normal pancreatic tissue thereby decreasing the risk of exocrine and endocrine impairment. ${ }^{7}$ Usually, patients with pancreatic neck tumors undergo distal pancreatectomy with or without splenectomy. Such extensive resection is associated with exocrine and endocrine long-term insufficiency with the reported incidence of developing diabetes mellitus is between 17 to $85 \% .^{2}$ The incidence of postoperative endocrine insufficiency following middle pancreatectomy has been reported to be as low as $4 \% .{ }^{8}$ Preservation of spleen is said to avoid infective and haematological consequences of splenectomy in these patients.

Most of the series on middle pancreatectomy have reported no mortality; one of the two patients in our series had surgery related death. ${ }^{2,8,9}$ In this patient GIA stapler was used to transect the proximal part of the pancreas which fractured the gland. Though, the proximal stump was reinforced by suturing, patient developed grade $\mathrm{C}$ pancreatic fistula from the proximal stump which leads to intraabdominal abscess, postpancreatectomy hemorrhage, sepsis and death. The main disadvantage of middle pancreatectomy is the risk of double pancreatic fistula. The incidence of pancreatic fistula is reported to be 14 to $30 \%$ as compared to 5 to $15 \%$ in distal pancreatectomy. ${ }^{6}$ Most series have no mortality but high morbidity following middle pancreatectomy, the mortality in our series was due to pancreatic fistula from the proximal stump where the stapler was used. The incidence of pancreatic fistula is reported to be high with the stapler closure. ${ }^{10}$

\section{Conclusions}

Though we had one death, we presume that it was due to the stapler and would have occurred even if we had performed distal pancreatectomy in that patient. Thus, it can be concluded that middle pancreatectomy can be recommended for benign or borderline tumours of the pancreatic neck as it is technically feasible and safe procedure.

\section{References}

1. Asanuma Y, Koyama K, Saito K, Tanaka J. An appraisal of segmental pancreatectomy for benign tumors of the 
pancreatic body: a report of two cases. Surg Today 1993; 23:733-6.

2. Bassi C. Middle segment pancreatectomy: A useful tool in the management of pancreatic neoplasm. J Gastrointest Surg 2007;11:421-4.

3. Letton AH, Wilson JP. Traumatic severance of pancreas treated by Roux-Y anastomosis. Surg Gynecol Obstet 1959; 109:473-8.

4. Iacono C, Bortolasi L, Facci E, Nifosi F, Pachera S, Ruzzenente A, et al. The Dagradi-Serio-Iacono operation central pancreatectomy. J Gastrointest Surg 2007; 11(3): 364-76.

5. Reber HA. Middle pancreatectomy: why I rarely do it. J Gastrointest Surg 2007; 11:730-2.

6. Iacono $\mathrm{C}$ et al. Is there a place for central pancreatectomy in pancreatic surgery? J Gastrointest Surg 1998; 2: 509-17.

7. Warshaw AL, Rattner DW, Fernandez-del Castillo, Z'graggen K. Middle segment pancreatectomy. A novel technique for conserving pancreatic tissue. Arch Surg. 1998; 133:327-31.

8. Roggin KK, Rudloff U, Blumgart LH, Brennan MF. Central pancreatectomy revisited. J Gastrointest Surg 2006; 10(6): 804-12.

9. Iacono C, Bortolasi L, Serio G. Indications and technique of central pancreatectomy - early and late results. Langenbecks Arch Surg. 2005; 390(3): 266-71.

10. Kleeff J, Diener MK, Zgraggen K, et al. Distal pancreatectomy: risk factors for surgical failure in 302 consecutive cases. Surg 2007; 245: 573-82. 